\title{
Novel AuPd bimetallic alloy decorated 2D BiVO 4 nanosheets with enhanced photocatalytic performance under visible light irradiation
}

\author{
Junlong Zhang ${ }^{a, b}$, Yan Lu $^{b}$, Lei Ge*a,b ${ }^{\text {, Changcun Han }}{ }^{\text {b }}$, Yujing Li ${ }^{\text {, }}$, \\ Yangqin $\mathrm{Gao}^{\mathrm{b}}$, Songsong $\mathrm{Li}^{\mathrm{b}}$, $\mathrm{Hao} \mathrm{Xu}^{\mathrm{b}}$
}

a State Key Laboratory of Heavy Oil Processing, College of Science, China University of Petroleum Beijing, No. 18 Fuxue Rd., Beijing 102249, People's Republic of China.

${ }^{\mathrm{b}}$ Department of Materials Science and Engineering, College of Science, China University of Petroleum Beijing, No. 18 Fuxue Rd., Beijing 102249, People's Republic of China.

\begin{abstract}
Novel AuPd bimetallic alloy nanoparticles (NPs) decorated two-dimensional (2D) $\mathrm{BiVO}_{4}$ nanosheet photocatalysts were successfully synthesized via mild wet-chemical process and facile deposition procedure. The composite photocatalysts were characterized by X-ray diffraction (XRD), scanning electron microscopy (SEM), transmission electronic microscope (TEM), X-ray photoelectron spectroscopy (XPS), UV-visible diffuse reflection spectroscopy (DRS), electron spin resonance (ESR) and surface photovoltage spectroscopy (SPV). The $1.0 \mathrm{wt} \% \mathrm{AuPd} / \mathrm{BiVO}_{4}$ nanosheets showed highest photocatalytic activity for the degradation of Rhodamine B (RhB) under visible light (300W Xe lamp coupled with a UV-cutoff filter $(\lambda \geq 400 \mathrm{~nm}))$, and the photocatalytic efficiency was improved for about 21.9 and 6.1 times as compared to pure $\mathrm{BiVO}_{4}$ and $1.0 \mathrm{wt} \% \mathrm{Au} / \mathrm{BiVO}_{4}$, respectively. The novel $\mathrm{AuPd} / \mathrm{BiVO}_{4}$ composites may make a way to synthesize 2D semiconductor composites with potential applications for light energy conversion and environmental purifications.
\end{abstract}

Keywords: 2D $\mathrm{BiVO}_{4}$ nanosheets; AuPd bimetallic alloy; Rhodamine B; Composite photocatalysts; Photocatalysis.

\footnotetext{
* Corresponding author at: State Key Laboratory of Heavy Oil Processing, China University of Petroleum Beijing, No. 18 Fuxue Road, Beijing102249, PR China. Tel/Fax.: +86 1089739096. E-mail address: gelei@cup.edu.cn （ L.Ge )
} 


\section{Introduction}

In the past few years, tremendous interest has been attracted by researchers related to photocatalytic pollutant degradation and water splitting, in which an ideal "green" technology known as semiconductor photocatalysis plays a vital role [1-4]. To develop more efficient photocatalysts with reasonable photocatalytic activities for practical applications, photocatalyst exhibits photocatalytic activity in visible range is required and so called "visible-light-active photocatalyst", such as $\mathrm{BiVO}_{4}$ [5,6], $\mathrm{Bi}_{2} \mathrm{WO}_{6}$ [7], $\mathrm{AgX}(\mathrm{AgCl}, \mathrm{AgBr})$ [8,9], $\mathrm{C}_{3} \mathrm{~N}_{4}$ [10] and CdS [11] have been developed and investigated as promising candidates to replace traditional non-visible-active photocatalyst, such as $\mathrm{TiO}_{2}$. With their intrinsic advantage to utilize solar energy covering the visible range, the research on visible-light-active photocatalysts with high stability is becoming more important with time.

Recently, $\mathrm{BiVO}_{4}$ has attracted much attention, which due to not only its potential application in photocatalytic water oxidation and degradation of hazardous organic pollutants, but also its good chemical stsbility under visible light irradiation [12, 13]. It has been shown that the photocatalytic activity of $\mathrm{BiVO}_{4}$ depends strongly on its crystalline form as well as microstructures. Generally, $\mathrm{BiVO}_{4}$ has three crystalline phases [14], among which the monoclinic scheelite $\mathrm{BiVO}_{4}\left(\mathrm{~m}-\mathrm{BiVO}_{4}\right)$ has a band gap of 2.4-2.5 eV and shows much higher photocatalytic activity than the other two forms. m- $\mathrm{BiVO}_{4}$ could be prepared into multifarious morphologies, such as nanosheets [5], nanoribbons [15], decahedrons [16], spheres [17], and hollow structure in olive shape [18]. Among them, we select two-dimensional nanosheets $\left(\mathrm{m}-\mathrm{BiVO}_{4}\right)$ as a support due to their large surface areas and quantum confinement effect arises from its thin sheet thickness in several nanometers

Besides of playing with the crystalline forms and microstructures, constructing composite structures is also proven to be an effective method to enhance the photocatalytic performance of 
m-BiVO present in pristine m-BiVO . Much effort has been spent to address the problem of low charge separation efficiency, such as combining $\mathrm{m}-\mathrm{BiVO}_{4}$ with metals $[6,19,20]$, metal oxides [21, 22], carbon materials [23] and silver compounds which showed improved photocatalytic performance, but is still under investigation for further improvement [24]. Metal nanoparticles, especially noble metals, such as $\mathrm{Au}, \mathrm{Ag}, \mathrm{Pd}$ and Pt, are studied as wonderful co-catalyst for photocatalysis. The low lying of Fermi levels in noble metals provides additional benefits in prolonging the lifetime of charge carriers to further improve the photocatalytic activity. Meanwhile, various bimetallic alloy NPs have been reported, such as AuPd [25], AuAg [26], PtCo [27] and PtCu [28] and exhibit superior activity than their corresponding monometallic NPs. Among them, AuPd NPs have been studied in multiple domains, including selective oxidation of primary carbon-hydrogen bonds [29], photocatalytic hydrogen evolution [30], electrocatalyst for ethanol oxidation [31] and phenol photodecomposition [32]. Although there are some literature have reported the photocatalytic performance of m-BiVO 4 and AuPd alloy respectively, but the $\mathrm{RhB}$ degradation performance of the $\mathrm{AuPd} / \mathrm{BiVO}_{4}$ composite which the AuPd NPs may be a promising candidate to construct composite structures with $\mathrm{m}-\mathrm{BiVO}_{4}$ to exhibit enhanced photocatalytic performance was not reported.

In this study, for the first time we proposed a facile method to prepare novel $\mathrm{AuPd} / \mathrm{BiVO} 4$ composites by in-situ depositing AuPd NPs on the surface of $2 \mathrm{D} \mathrm{m}-\mathrm{BiVO}_{4}$ nanosheets. The photocatalytic activity was investigated by degradation of Rhodamine B (RhB) under visible light irradiation and reconfirmed with degradation of phenol. The result indicated that the as-prepared $\mathrm{AuPd} / \mathrm{BiVO}_{4}$ nanosheets showed significantly enhanced photocatalytic activities than pure $\mathrm{BiVO}_{4}$. The active photocatalytic species and dynamic behavior of photo-generated charge carriers was investigated via ESR and SPV techniques. A possible photocatalytic mechanism was proposed to illustrate the 
separation and transfer behaviors of photo-generated electron-hole pairs in the AuPd/BiVO $\mathrm{C}_{4}$ The successful development of the novel $\mathrm{AuPd} / \mathrm{BiVO}_{4}$ nanosheets with enhanced photocatalytic activity and acceptable stability may pave a way to synthesize 2D semiconductor composites for potential applications in light energy conversion and environmental purifications.

\section{Experimental}

\subsection{Materials}

Bismuth nitrate pentahydrate $\left(\mathrm{Bi}\left(\mathrm{NO}_{3}\right)_{3} \cdot 5 \mathrm{H}_{2} \mathrm{O}\right.$, Aladdin, 98\% A.C.S), ammonium metavanadate ( $\mathrm{NH}_{4} \mathrm{VO}_{3}$, A.R.), sodium dodecylbenzenesulfonate $\left(\mathrm{C}_{18} \mathrm{H}_{29} \mathrm{NaO}_{3} \mathrm{~S}\right.$, A.R.), sodium hydroxide ( $\mathrm{NaOH}$, A.R.), nitric acid $\left(\mathrm{HNO}_{3}, 65 \%\right)$, Sodium borohydride $\left(\mathrm{NaBH}_{4}\right.$, Aladdin, 98\%), poly vinyl alcohol (PVA, Aldrich, MW = 10 000, 80\% hydrolyzed), ethyl alcohol (EtOH, A.R.) and Rhodamine B (RhB, A.R.) was used as purchased without further purification. Deionized water was used as the solvent for all of the solutions or dispersions.

\subsection{Synthesis of the $\mathrm{BiVO}_{4}$ nanosheets}

The $\mathrm{BiVO}_{4}$ nanosheets were prepared according to reference [5] with slight modification. In a typical procedure, $1 \mathrm{mmol} \mathrm{Bi}\left(\mathrm{NO}_{3}\right)_{3} \cdot 5 \mathrm{H}_{2} \mathrm{O}$ and $0.72 \mathrm{mmol} \mathrm{C}_{18} \mathrm{H}_{29} \mathrm{NaO}_{3} \mathrm{~S}$ (SDBS) were initially dissolved in $10.0 \mathrm{~mL} 4.0 \mathrm{~mol} / \mathrm{L} \mathrm{HNO}_{3}$ solution and the resultant solution was marked as solution A. Simultaneously, $1.0 \mathrm{mmol} \mathrm{NH} \mathrm{VO}_{3}$ was added into $10.0 \mathrm{~mL} 2.0 \mathrm{~mol} / \mathrm{L} \mathrm{NaOH}$ solution, and the resultant solution was marked as solution B. Then, solution B was added into solution A under vigorous stirring. The $\mathrm{pH}$ of the mixed solution was adjusted to 6.5 with $2 \mathrm{M} \mathrm{NaOH}$ solution. The resultant solution was sealed in Teflon-lined stainless steel autoclave which was heated at $160^{\circ} \mathrm{C}$ for 3 h. The precipitate (vivid yellow) was collected by centrifugation and rinsed with distilled water and absolute alcohol and then dried at $100^{\circ} \mathrm{C}$ for $4 \mathrm{~h}$

\subsection{Synthesis of AuPd/BiVO}


The AuPd alloy NPs were prepared as follow [25, 33]: $10 \mathrm{mM} \mathrm{HAuCl} 4 \cdot 3 \mathrm{H}_{2} \mathrm{O}$ and $\mathrm{PdCl}_{2}$ aqueous solution were prepared as stock solutions. Fresh aqueous solutions of $0.1 \mathrm{M} \mathrm{NaBH}_{4}$ and $1.0 \mathrm{wt} \%$ poly vinyl alcohol (PVA) were also prepared. The prepared $\mathrm{BiVO}_{4}$ nanosheets $(0.1 \mathrm{~g})$ were dispersed in 50 $\mathrm{mL}$ deionized water to form homogeneous suspension under continuous stirring. Then the $\mathrm{PdCl}_{2}$ and $\mathrm{HAuCl}_{4} \cdot 3 \mathrm{H}_{2} \mathrm{O}$ stock solution was mixed in desired ratio $(\mathrm{Au} / \mathrm{Pd}=1$, weight ratio) and an appropriate amount of PVA solution was added $\left(\mathrm{PVA} /(\mathrm{Au}+\mathrm{Pd})=1.2\right.$, weight ratio). After that, the fresh $\mathrm{NaBH}_{4}$ solution $\left(\mathrm{NaBH}_{4} /(\mathrm{Au}+\mathrm{Pd})=5\right.$, molar ratio) was added and a dark green solution was obtained. After stirring for $2 \mathrm{~h}$ at room temperature, the products were purified and collected, then dried at $80^{\circ} \mathrm{C}$. With the same procedure, $\mathrm{AuPd} / \mathrm{BiVO}_{4}$ with different weight ratio of AuPd bimetallic NPs was obtained by changing the weight contents of AuPd precursors in the mixed solution. $1.0 \mathrm{wt} \% \mathrm{Au} / \mathrm{BiVO}_{4}$ and 1.0 wt\% $\mathrm{Pd} / \mathrm{BiVO}_{4}$ was also synthesized for comparison by using the same procedure with pure Au or Pd precursors.

\subsection{Characterization}

The crystal structure of the samples was analyzed by X-ray diffraction (XRD; Bruker D8 Advance, $\mathrm{X}$-ray diffractometer) with $\mathrm{CuK} \alpha$ radiation at a scan step size of $3^{\circ}$. The morphology of the samples was examined by field emission canning electronic microscope (FESEM, FEI Quanta 200F) and High resolution transmission electron microscopy (HRTEM). UV-vis diffuse reflection spectroscopy (DRS) was performed on a Shimadzu UV-4100 spectrophotometer using $\mathrm{BaSO}_{4}$ as the reference material. The X-ray photoelectron spectroscopy (XPS) was measured on a PHI 5300 ESCA system with a beam voltage of $3.0 \mathrm{eV}$, and the energy of $\mathrm{Ar}$ ion beam was $1.0 \mathrm{keV}$ to detect the composition of the final products. The binding energies were normalized to the signal for adventitious carbon at $284.8 \mathrm{eV}$. The electron spin resonance (ESR) signals of spin-trapped oxidative radicals were obtained on a Bruker model ESR JES-FA200 spectrometer equipped with a quanta-Ray Nd:YAG laser system as the light 
source, in which a UV-cutoff filter (kP400 nm) was used.

\subsection{SPV measurements}

The surface photovoltage (SPV) measurement was carried out on a surface photovoltage spectroscopy (PL-SPS/IPCE1000 Beijing Perfect Light Technology Co., Ltd). The measurement system consists of a source of monochromatic light, a locking amplifier (SR830, Stanford research systems, Inc.) with a light chopper (SR540, Stanford research systems, Inc.), and a simple chamber. The monochromatic light is provided by passing light from a $500 \mathrm{~W}$ xenon lamp (CHFXQ500 W, global xenon lamp power) though a grating mono-chromator (Omni-5007, No.09010, Zolix), which chopped with a frequency of $24 \mathrm{~Hz}$. All the measurements were operated under ambient pressure at room temperature.

\subsection{Photocatalytic activity}

The photocatalytic activities of the as-prepared samples were investigated by degradation of RhB under visible-light irradiation. In a typical photocatalytic experiment, 0.05 g photocatalyst powder was suspended in $100 \mathrm{ml} \mathrm{RhB}$ aqueous solution $(10 \mathrm{mg} / \mathrm{L})$. The suspension was stirred in the dark for $1 \mathrm{~h}$ to establish adsorption-desorption equilibrium. After that, 300W Xe lamp coupled with a UV-cutoff filter $(\lambda \geq 400 \mathrm{~nm})$ was used as the visible light source to irradiate the suspensions under vigorous stirring. The light intensity employed was $72 \mathrm{~mW} \cdot \mathrm{cm}^{-2}$. After irradiation, $4 \mathrm{~mL}$ sample solution was collected at certain time interval, the photocatalysts were removed by centrifugation, and the remaining solution was analyzed by the absorption of $\mathrm{RhB}$ at 552nm using UV-1700 spectrometer.

The photodegradation of colorless phenol was also performed. In the phenol degradation process, $50 \mathrm{mg}$ photocatalyst powder was suspended in $100 \mathrm{ml}$ phenol solution $(20 \mathrm{mg} / \mathrm{L})$, other steps are the same as RhB degradation process. The solution was collected in certain intervals and centrifuged, and phenol absorption in the collected liquid was then analyzed by UV -1700 spectrometer at $270 \mathrm{~nm}$ 
(quartz cuvette).

\section{Results and discussion}

\subsection{Characterization of $\mathrm{AuPd} / \mathrm{BiVO}_{4}$ composites}

The crystal structure and phase purity of as-prepared products were first verified by XRD analysis. The representative XRD patterns of $\mathrm{BiVO}_{4}$ nanosheets and $\mathrm{BiVO}_{4}$ nanosheet cocatalyzed with noble metals are shown in Fig. 1, data of the standard card of monoclinic $\mathrm{BiVO}_{4}$ (JCPDS No. 14-0688) are also shown as reference. Well correlation of main peak positions can be seen as compare the $\mathrm{BiVO}_{4}$ nanosheets diffraction pattern with the reference, indicating the $\mathrm{BiVO}_{4}$ nanosheets is mainly composed by monoclinic $\mathrm{BiVO}_{4}$ with little impurity. The main peaks at $2 \theta$ of $18.99^{\circ}, 28.82^{\circ}, 30.55^{\circ}, 42.46^{\circ}, 47.31^{\circ}$ and $53.31^{\circ}$ can be readily indexed to the (011), (1 $\left.\overline{\mathbf{z}}^{1}\right),(040),(051),(042)$ and (161) planes of monoclinic $\mathrm{BiVO}_{4}$, respectively. Clearly, the $\mathrm{AuPd} / \mathrm{BiVO}_{4}$ composites have similar XRD patterns as the $\mathrm{BiVO}_{4}$ nanosheets with indistinguishable difference, indicates the $\mathrm{BiVO}_{4}$ crystal structure keeps unchanged during the reduction of noble metal precursors to metal NPs in $\mathrm{NaBH}_{4}$ solution. No typical

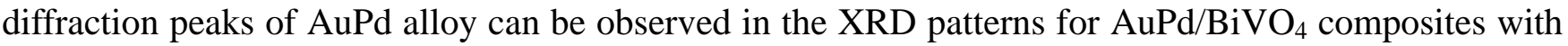
different weight ratio of AuPd NPs, which may due to the low contents of AuPd in the $\mathrm{AuPd} / \mathrm{BiVO} 4$ composites.

To confirm the successful loading of AuPd NPs on the $\mathrm{BiVO}_{4}$ nanosheets, elemental and microstructure morphology analysis was performed by HRTEM and SEM characterization. Fig. 2 presents typical TEM and SEM images of $\mathrm{AuPd} / \mathrm{BiVO}_{4}$ composite with $\mathrm{X} \%$ loading of AuPd NPs. The SEM image shows the pure $\mathrm{BiVO}_{4}$ sample has a well-defined nanosheet structure with smooth surface (Fig. 2a). Rough surface is shown in $\mathrm{AuPd} / \mathrm{BiVO}_{4}$ composite (Fig. 2b), indicating AuPd NPs are successfully loaded on the $\mathrm{BiVO}_{4}$ nanosheets. The small NPs appeared on the rough surface of AuPd/BiVO ${ }_{4}$ composite can be assigned to surface loaded AuPd NPs. A close TEM view of the 
AuPd/BiVO 4 composite (Fig. 2c) reveals the AuPd NPs are uniformly distributed on the surface of $\mathrm{BiVO}_{4}$ nanosheets with an average size of $\sim 7 \mathrm{~nm}$. A representative HRTEM image which taken from a selected area of the $\mathrm{AuPd} / \mathrm{BiVO}_{4}$ composite structure is shown in Fig. 2d. The spacing of the lattice fringe is found to be about 2.33nm can be well indexed as (111) plane of the AuPd nanoparticles, which confirms the surface loaded NPs are AuPd NPs [33]. The intimate interfaces formed between AuPd NPs and $\mathrm{BiVO}_{4}$ nanosheets as viewed in HRTEM image is favorable for charge separation and transfer of charge carriers. The signal of Au and Pd shown in EDX pattern (Fig. 2e) further confirm the presence of AuPd in the composites. Fig. 3(a) shows a TEM image of AuPd/BiVO 4 at low magnification. Fig. 3(b-f) displays the elemental distribution maps $(\mathrm{O}, \mathrm{Pd}, \mathrm{Au}, \mathrm{V}$ and $\mathrm{Bi}$ respectively) determined by energy-filtered TEM technique through the nanostructure in Fig. 3(a). The result of $\mathrm{AuPd} / \mathrm{BiVO}_{4}$ shows that elements of $\mathrm{Bi}, \mathrm{V}, \mathrm{O}, \mathrm{Au}$ and $\mathrm{Pd}$ can be clearly observed. It is found that $\mathrm{Bi}, \mathrm{V}, \mathrm{O}, \mathrm{Au}$ and $\mathrm{Pd}$ elements distribute almost uniformly in the surface of $\mathrm{AuPd} / \mathrm{BiVO}_{4}$, which further confirmed the $\mathrm{AuPd} / \mathrm{BiVO}_{4}$ composite photocatalysts were prepared successfully.

The UV-visible diffuse reflectance spectra of $\mathrm{AuPd} / \mathrm{BiVO}_{4}$ composites with different weight ratio of AuPd NPs, together with that of $\mathrm{BiVO}_{4}$ nanosheets were investigated and shown in Fig. 4. The $\mathrm{BiVO}_{4}$ nanosheets have absorption from the UV range up to about $517 \mathrm{~nm}$ in the visible range, and the red tangent line of spectrum denotes that the visible light absorption is ascribed to the band gap transition, in accordance with the reported band gap of $\sim 2.4 \mathrm{eV}$ for $\mathrm{BiVO}_{4}$. With the introduction of AuPd alloy nanoparticles, the colors of $\mathrm{AuPd} / \mathrm{BiVO}_{4}$ samples are changed from yellow to dark grey. The AuPd/BiVO 4 composites display similar absorption edge after introduction of different weight ratio of AuPd NPs, but exhibit significantly enhanced absorption in visible range beyond the optical response of $\mathrm{BiVO}_{4}$. The increase in visible light absorption with higher AuPd NPs weight ratio clearly demonstrate that the enhanced light absorption beyond the optical response of $\mathrm{BiVO}_{4}$ for $\mathrm{AuPd} / \mathrm{BiVO} 4$ 
arises from the absorption of AuPd NPs, which confirms that the introduction of AuPd NPs could improve the visible light absorption of $\mathrm{BiVO}_{4}$. It is worth to note that enhanced light absorption usually accompany with improved photocatalytic performance.

The surface element composition and atomic valence state of $\mathrm{AuPd} / \mathrm{BiVO}_{4}$ sample with $1.0 \mathrm{wt} \%$ AuPd was examined by X-ray photoelectron spectroscopy (XPS). In Fig. 5a, the two peaks fitted at 158.4 and $163.7 \mathrm{eV}$ are the signals of $\mathrm{Bi} 4 \mathrm{f}_{7 / 2}$ and $\mathrm{Bi} 4 \mathrm{f}_{5 / 2}$, which are less than that of $158.9 \mathrm{eV}$ and 164.3 eV of pure $\mathrm{BiVO}_{4}$ [34]. This phenomenon indicates that there may have an interaction on the interface between AuPd NPs and $\mathrm{BiVO}_{4}$ nanosheets. The V 2p peaks located at 516.3 and $523.8 \mathrm{eV}$ are in accordance with the $\mathrm{V} 2 \mathrm{p}_{3 / 2}$ and $\mathrm{V} 2 \mathrm{p}_{1 / 2}$ in the $\mathrm{BiVO}_{4}$ (Fig. $5 \mathrm{~b}$ ). It can be clearly observed that $\mathrm{O} 1 \mathrm{~s}$ spectra can be fitted into two peaks, representing the presence of different oxygen species on the sample surface, including lattice oxide $\left(\mathrm{O}_{\mathrm{I}}\right)$ species $(529.3 \mathrm{eV})$ and adsorbed oxygen $\left(\mathrm{O}_{\mathrm{II}}\right)$ species $(531.2 \mathrm{eV})$ in Fig. 5c [35]. The Au 4f XPS spectra (Fig. 5d) shows that two peaks at 82.66 and 86.35 eV can be assigned to $4 \mathrm{f}_{7 / 2}$ and $4 \mathrm{f}_{5 / 2}$ of $\mathrm{Au}^{0}$ species, which exhibits slight negative shift as compared to previous report [36]. The shift may be due to the unique structure of AuPd bimetallic alloys. As shown in Fig. 5e, two asymmetric peaks which located at 334.3 and $339.8 \mathrm{eV}$ are the signals of $\mathrm{Pd} 3 \mathrm{~d}_{5 / 2}$ and $\mathrm{Pd} 3 \mathrm{~d}_{3 / 2}$, respectively. It is attributed to the metallic $\mathrm{Pd}^{0}$ species. However, the binding energy peak centered at 335.1 and $340.1 \mathrm{eV}$ are originates from of $\mathrm{Pd}^{2+}$ species. The result is in accordance with our previous work [25], and may be ascribed to the charge transfer between Au and Pd in AuPd bimetallic alloy.

\subsection{Visible light photocatalytic activity of $\mathrm{AuPd} / \mathrm{BiVO}_{4}$ samples}

There are lots of documents studied the photoactivity of $\mathrm{BiVO}_{4}$-based photocatalysts through degradation of $\mathrm{RhB}$. Li et al. prepared $\mathrm{BiVO}_{4} / \mathrm{BiOBr}$ composite by a simple one-pot hydrothermal process which the RhB (50 ml, $10 \mathrm{mg} / \mathrm{L}$ ) was completely degraded in 15 min under the illumination of visible light $(\lambda>420 \mathrm{~nm})$ [37]. The Z-scheme $\mathrm{CdS} / \mathrm{BiVO}_{4} \mathrm{NWs}$ with different weight ratios were 
synthesized by Zhou et al., in which the $1: 2 \mathrm{CdS} / \mathrm{BiVO}_{4}$ could complete degradation of $\mathrm{RhB}$ (50 ml, $\left.2 \times 10^{-5} \mathrm{M}\right)$ only took 35 min under visible light $(\lambda>420 \mathrm{~nm})$ [38]. Yu et al. prepared $\mathrm{RGO} / \mathrm{BiVO}_{4}$ composite through an in-situ hydrothermal-assisted growth strategy which $10 \mathrm{mg} / \mathrm{L} \mathrm{RhB}$ solution was completely degraded in $8 \mathrm{~h}$ under natural sunlight [39]. The photocatalytic activity of the as-prepared samples was studied by the degradation of RhB under visible light irradiation ( $\lambda \geq 400 \mathrm{~nm})$. As compared to the as-prepared $\mathrm{BiVO}_{4}$ nanosheets, all of the co-catalyzed samples with surface decoration of AuPd, Au and Pd NPs exhibit significantly enhanced photocatalytic activity (Fig. 6). Interestingly, for the samples with the same loading amount of co-catalyst, the $\mathrm{AuPd} / \mathrm{BiVO}_{4}$ sample shows higher photocatalytic activity than the samples decorated by pure Au or Pd NPs, which reveals a superior photocatalytic activity enhancement of AuPd NPs as compared to pure Au and Pd NPs. This superior photocatalytic activity enhancement for $\mathrm{AuPd} / \mathrm{BiVO}_{4}$ sample can be attributed to the formation of intimate interfaces between AuPd NPs and $\mathrm{BiVO}_{4}$ nanosheets, in turns the charge separation efficiency of photo-excited charge carriers is effectively promoted. The photocatalytic activity of $\mathrm{AuPd} / \mathrm{BiVO}_{4}$ sample shows an increase at the initial stage with increasing the AuPd NPs weight ratio, reaching the highest point at $1 \mathrm{wt} \%$ loading of AuPd NPs, and then declines with further loading of AuPd NPs. This decline in photocatalytic activity with further loading of AuPd NPs can be related to the increase in the opacity of the composite samples. Furthermore, introduction of higher content of AuPd NPs would also lead to active site covering on the surface of $\mathrm{BiVO}_{4}$, which hinders the migration of charge carriers at the semincoductor/electrolyte interface. These results indicate that appropriate loading of AuPd NPs on $\mathrm{BiVO}_{4}$ nanosheets indeed contribute to improve the photocatalytic activity toward the degradation of organic dyes under visible light.

To further quantitatively investigate the reaction kinetics of the RhB photodegradation, the RhB photodegradation data was fitted by applying a first-order model [40]. The corresponding $\ln \left(\mathrm{C}_{0} / \mathrm{C}\right)$ plot 
is shown in Fig. 7a and the fitted degradation rate constant of RhB is illustrated in Fig. 7b. It is obvious that the $1.0 \mathrm{wt} \% \mathrm{AuPd} / \mathrm{BiVO}_{4}(\mathrm{~A}-1)$ sample has the maximum degradation rate constant, and is about 21.9, 6.1 and 1.2 times higher than the pure $\mathrm{BiVO}_{4}(\mathrm{~A}), 1.0 \mathrm{wt} \% \mathrm{Au} / \mathrm{BiVO}_{4}(\mathrm{~A}-6)$ and $1.0 \mathrm{wt} \%$ $\mathrm{Pd} / \mathrm{BiVO}_{4}(\mathrm{~A}-5)$, respectively. The high catalytic performance can be attributed to the formation of the formation of intimate interfaces between AuPd NPs and $\mathrm{BiVO}_{4}$ nanosheets as mentioned before, which are favorable of transfer and separation of the photo-excited charge carriers generated in $\mathrm{BiVO}_{4}$.

The RhB dye photosensitization effect may exist in the reaction process, and the dye sensitization makes the photodegradation of RhB more easily. In order to eliminate the phenomenon caused by the dye sensitization, degradation of colorless organic phenol was also investigated to further verify the photocatalytic ability of $1.0 \mathrm{wt} \% \mathrm{AuPd} / \mathrm{BiVO}_{4}$. The photodecomposition of phenol is slower than $\mathrm{RhB}$, but still reached 56\% degradation within $2 \mathrm{~h}$ under visible light irradiation (Fig. 8). The low performance for photodecomposition of phenol is due to the concentrated solution used in the experiment, which is twice higher than the one used in photodegradation of $\mathrm{RhB}$, and also because the stable benzene ring exist in the phenol molecule, which is difficult to decompose. To some extent, the result confirms that the as-prepared $\mathrm{AuPd} / \mathrm{BiVO}_{4}$ has photocatalytic ability and can effectively degrade organic molecules.

The stability of a photocatalyst is a critical attribute for practical application. Cycling runs for the photo-oxidation of $\mathrm{RhB}$ with $1.0 \mathrm{wt} \% \mathrm{AuPd} / \mathrm{BiVO}_{4}(\mathrm{~A}-1)$ was performed to evaluate its photocatalytic stability. After every 25 min of photodegradation process, the sample was recycled by centrifugation, washed with distilled water, and reused in next cycle. Fig. 9 shows that the photocatalytic activity of 1.0 wt $\% \mathrm{AuPd} / \mathrm{BiVO}_{4}$ still conserve $75 \%$ of its original activity after being recycled for four times, which indicates a reliable stability. The photocatalytic stability of $1.0 \mathrm{wt} \% \mathrm{AuPd} / \mathrm{BiVO}_{4}$ can be even higher when considering the loss of the photocatalyst during the centrifugation process.

\subsection{Photocatalytic mechanism discussion}


To investigate the photocatalytic mechanism for $\mathrm{AuPd} / \mathrm{BiVO}_{4}$ composites, the ESR and SPV techniques were performed. It is generally accepted that organic pollutants can be degraded by photocatalytic oxidation processes. In this procedure, a series of photo-induced reactive species, such as $\mathrm{h}^{+}, \mathrm{OH}$, and $\cdot \mathrm{O}_{2}^{-}$, are suspected to be involved in the photocatalytic degradation reaction. Free radicals in reaction systems are generally detected by means of ESR technique. In ESR test, DMPO (5, 5-dimethyl-1-dimethy-N-oxide) is often used as a radical scavenger, resulting in formation of detectable stable free radicals DMPO-· ${ }_{2}{ }^{-}$or DMPO-·OH. Fig. 10 shows ESR spectra measured with/without light irradiation over the $1.0 \mathrm{wt} \% \mathrm{AuPd} / \mathrm{BiVO}_{4}$ photocatalyst with different solvents at room temperature and air atmosphere. No ESR signal can be seen under dark condition. On the contrary, a gradual evolution of ESR peaks for DMPO- $\cdot \mathrm{O}_{2}{ }^{-}$adducts (six characteristic peaks, red line) can be observed under visible light irradiation (Fig. 10a). However, no signals of DMPO-·OH adducts were detected both in dark and illuminated conditions (Fig.10b), which means that $\cdot \mathrm{O}_{2}{ }^{-}$is one of the main active species involved in the photodegradation process.

To discover the role of photo-generated holes in the photodegradation process, $0.05 \mathrm{~g}$ KI served as scavenger for $\mathrm{h}^{+}$and $\cdot \mathrm{OH}$ species was added into the mixed solution, and the same photodegradation of $\mathrm{RhB}$ experiment was repeated. It is shown that the degradation activity of $1.0 \mathrm{wt} \% \mathrm{AuPd} / \mathrm{BiVO}_{4}$ decreased rapidly after addition of KI (Fig. 11), which should be caused by the hole trapping of KI. Therefore, we can conclude that the photo-generated holes are another active species involved in the photodegradation process.

The dynamic behavior of the photo-generated charge carriers was also investigated by using surface photovoltage spectroscopy (SPV) (Fig. 12). Pure $\mathrm{BiVO}_{4}$ nanosheets present a clear positive SPV signal after being excited by light with wavelength ranging from $300-525 \mathrm{~nm}$, which is a typical characteristic of n-type semiconductor in SPV. The positive SPV signal indicates that photo-excited 
positive charges are transferred from inner semiconductor to the surface. The composite samples exhibit stronger SPV signal than pure $\mathrm{BiVO}_{4}$ nanosheets, indicating that the recombination of electron-hole pairs is effectively inhibited. The $1.0 \mathrm{wt} \% \mathrm{AuPd} / \mathrm{BiVO}_{4}$ sample shows the highest SPV intensity, in turns most efficient charge separation efficiency, which is also consistent with the results of photocatalytic experiment and well explained the origin of enhanced catalytic performance in the $\mathrm{AuPd} / \mathrm{BiVO}_{4}$ composites. The enhanced separation efficiency of electron-hole pairs can be attributed to the efficient charge transfer at the interface formed between AuPd NPs and $\mathrm{BiVO}_{4}$ nanosheets in the composite samples.

A possible photocatalytic mechanism in $\mathrm{AuPd} / \mathrm{BiVO}_{4}$ sample is proposed based on the above observations (as shown in Fig. 13). In general, photo-generated electrons and holes in pristine $\mathrm{BiVO}_{4}$ are likely to recombine after being excited by incident photons. After introducing of AuPd bimetallic NPs, intimate contacts are formed at the AuPd NPs/BiVO 4 interfaces and serve as effective channels for the flow of photo-generated charge carriers. As AuPd alloy possesses higher electron-capturing ability due to its low lying of Fermi level, electrons would be stimulated to transfer from $\mathrm{BiVO}_{4}$ to AuPd NPs, results in efficient charge separation efficiency. This improved charge separation efficiency facilitated at the AuPd NPs/BiVO 4 interface is the key point responsible for the enhancement of photocatalytic activity. Subsequently, the absorbed $\mathrm{O}_{2}$ trap electrons to form $\cdot \mathrm{O}_{2}{ }^{-}$, and holes transfer to the surface of $\mathrm{BiVO}_{4}$. Both $\cdot \mathrm{O}_{2}{ }^{-}$and $\mathrm{h}^{+}$serve as active species to oxidize organic pollutants so that the as-prepared $\mathrm{AuPd} / \mathrm{BiVO}_{4}$ sample exhibit superior photocatalytic activity.

\section{Conclusions}

In summary, novel AuPd bimetallic NPs decorated 2D $\mathrm{BiVO}_{4}$ nanosheets have been successfully synthesized via mild wet-chemical process and facile deposition process. The AuPd NPs dispersed uniformly on the surface of $\mathrm{BiVO}_{4}$ nanosheets and formed intimate interfaces. The 1.0 wt\% 
AuPd/BiVO 4 sample exhibited highest activity, which was about 21.9 times higher than pure $\mathrm{BiVO}_{4}$. The enhancement can be attributed to the efficient interfacial charge transfer in the composite sample. The ESR and radical capture experiments confirmed that $\cdot \mathrm{O}_{2}{ }^{-}$and $\mathrm{h}^{+}$were the active species in the degradation progress. The SPV measurement reveals that decoration of $2 \mathrm{D} \mathrm{BiVO}_{4}$ nanosheets with AuPd NPs facilitates charge separation in the composite structure. In conclusion, the $\mathrm{AuPd} / \mathrm{BiVO}_{4}$ composites are promising materials in photocatalytic organic degradation process, and our work may provide valuable information for modifying n-type semiconductor photocatalys with improved photocatalytic performance.

\section{Corresponding Author}

*E-mail: gelei@cup.edu.cn (Professor Lei Ge)

\section{Acknowledgements}

This work was financially supported by the National Science Foundation of China (Grant No. 21003157, 21273285 and 51572295), Beijing Nova Program (Grant No. 2008B76), and Science Foundation of China University of Petroleum, Beijing (Grant No. KYJJ2012-06-20).

\section{References}

[1] X. F. Feng, S. Maier, M. Salmeron, Journal of the American Chemical Society 134 (2012) $5662-5668$.

[2] M.R. Hoffmann, S.T. Martin, W. Choi, D.W. Bahnemann, Chemical Reviews 95 (1995) 69-96.

[3] Kudo, Y. Miseki, Chemical Society Reviews 38 (2009) 253-278.

[4] Y. Zhang, Z.R. Tang, X. Fu, Y.J. Xu, ACS Nano 4 (2010) 7303-7314.

[5] Q. Yu, Z.R. Tang, Y.J. Xu, Journal of Energy Chemistry 23 (2014) 564-574. 
[6] S.W. Cao, Z. Yin, J. Barber, F.Y.C. Boey, S.C.J. Loo, C. Xue, ACS Applied Materials \& Interfaces 4 (2012) 418-423.

[7] L. Ge, C. C. Han, J. Liu, Applied Catalysis B: Environmental 108-109 (2011) 100-107.

[8] C. C. Han, L. Ge, C. F. Chen, Y. J. Li, Z. Zhao, X. L. Xiao, Z. L. Li, J. L. Zhang, Journal of Materials Chemistry A 2 (2014) 12594-12600.

[9] L. Zhang, K. H. Wong, Z. Chen, J. C. Yu, J. Zhao, C. Hu, C. Y. Chan, P. K. Wong, Applied Catalysis A: General 363 (2009) 221-229.

[10]F. Dong, Z. W. Zhao, T. Xiong, Z. L.Ni, W. D. Zhang, Y. J. Sun, W. K. Ho, ACS Applied Materials \& Interfaces 5 (2013) 11392-11401.

[11]Y. Shi, H. Y. Li, L. Wang, W. Shen, H. Z. Chen, ACS Applied Materials \& Interfaces 4 (2012) 4800-4806.

[12]L. Zhang, D. Chen, X. Jiao, The Journal of Physical Chemistry B 110 (2006) 2668-2673.

[13]Y. Sun, B. Qu, Q. Liu, S. Gao, Z. Yan, W. Yan, B. Pan, S. Wei, Y. Xie, Nanoscale 4 (2012) 3761-3767.

[14]L. Zhang, D. R. Chen, X. L. Jiao, The Journal of Physical Chemistry B 110 (2006) 2668-2673.

[15]F. X. Wang, M. W. Shao, L. Cheng, J. Hua, X. W. Wei, Materials Research Bulletin 44 (2009) 1687-1691.

[16]D. E. Wang, R. G. Li, J. Zhu, J. Y. Shi, J. F. Han, X. Zong, C. Li, The Journal of Physical Chemistry C 116 (2012) 5082-5089.

[17]W. Z. Yin, W. Z. Wang, L. Zhou, S. M. Sun, L. Zhang, Journal of Hazardous Materials 173 (2010) 194-199.

[18] M.L. Guan, D.K. Ma, S.W. Hu, Y.J. Chen, S.M. Huang, Inorganic Chemistry 50 (2011) 800-805. [19]L. Ge, Materials Chemistry and Physics 107 (2008) 465-470. 
[20]P. Zhang, J. Z. Zhang, Applied Surface Science 256 (2010) 3224-3227.

[21] W. Z. Wang, X. W. Huang, S. Wu, Y. X. Zhou, L. J. Wang, H. L. Shi, Y. J. Liang, B. Zou, Applied Catalysis B: Environmental 134-135 (2013) 293-301.

[22] S.J. Hong, S. Lee, J.S. Jang, J.S. Lee, Energy \& Environmental Science 4 (2011) 1781-1787.

[23] M. Ou, Q. Zhong, S. L. Zhang, L. M. Yu, Journal of Alloys and Compounds 626 (2015) 401-409.

[24]H. Y. Li, Y. J. Sun, B. Cai, S. Y. Gan, D. X. Han, L. Niu, T. S. Wu, Applied Catalysis B: Environmental 170-171 (2015) 206-214.

[25]C. C. Han, L. Wu, L. E. Ge, Y. J. Li, Z. Zhao, Carbon 92 (2015) 31-40.

[26] N. Zhou, L. Polavarapu, N. Y. Gao, Y. L. Pan, P. Y. Yuan, Q. Wang, Q.H. Xu, Nanoscale 5 (2013) 4236-4241.

[27]Y. Y. Jia, Y. Q. Jiang, J. W. Zhang, L. Zhang, Q. L. Chen, Z. X. Xie, L. S. Zheng, Journal of the American Chemical Society 136 (2014) 3748-3751.

[28]Z. F. Hu, J.C. Yu, Journal of Materials Chemistry A 1 (2013) 12221-12228.

[29]L. Kesavan, R. Tiruvalam, M.H.A. Rahim, M.I. bin Saiman, D.I. Enache, R.L. Jenkins, N. Dimitratos, J.A. Lopez-Sanchez, S.H. Taylor, D.W. Knight, C.J. Kiely, G.J. Hutchings, Science 331 (2011) 195-199.

[30] Y. J. Xin, L. E. Wu, L. Ge, C. C. Han, Y. J. Li, S. M. Fang, Journal of Materials Chemistry A 3 (2015) 8659-8666.

[31]M. Nie, H. L. Tang, Z. D. Wei, S.P. Jiang, P.K. Shen, Electrochemistry Communications 9 (2007) 2375-2379.

[32]R. Su, R. Tiruvalam, Q. He, N. Dimitratos, L. Kesavan, C. Hammond, J.A. Lopez-Sanchez, R. Bechstein, C.J. Kiely, G.J. Hutchings, F. Besenbacher, ACS Nano 6 (2012) 6284-6292.

[33] Y. Lu, J. Zhang, L. Ge, C. Han, P. Qiu, S. Fang, J Colloid Interf. Sci. 483 (2016) 146-153. 
[34]M. C. Long, W. M. Cai, J. Cai, B. X. Zhou, X. Y. Chai, Y. H. Wu, The Journal of Physical Chemistry B 110 (2006) 20211-20216.

[35] J. Zhang, H. Cui, B. Wang, C. Li, J. P. Zhai, Q. Li, Chemical Engineering Journal 223 (2013) 737-746.

[36] A. Cybula, J. B. Priebe, M. M. Pohl, J. W. Sobczak, M. Schneider, A. Zielińska-Jurek, A. Brückner and A. Zaleska, Applied Catalysis B: Environmental 152-153 (2014) 202-211.

[37]W. Li, Y. Zhang, Y. Bu, Z. Chen, J Alloy. Compd. 680 (2016) 677-684.

[38]F.Q. Zhou, J.C. Fan, Q.J. Xu, Y.L. Min, Applied Catalysis B: Environmental 201 (2017) 77-83.

[39]C. Yu, S. Dong, J. Zhao, X. Han, J. Wang, J. Sun, J Alloy. Compd. 677 (2016) 219-227.

[40] M. Shang, W. Z. Wang, S. M. Sun, J. Ren, L. Zhou, L. Zhang, The Journal of Physical Chemistry C 113 (2009) 20228-20233. 


\section{Figure Captions:}

Fig. 1. XRD patterns of pure $\mathrm{BiVO}_{4}$ nanosheets, $\mathrm{Au} / \mathrm{BiVO} 4, \mathrm{Pd} / \mathrm{BiVO}_{4}$ and $\mathrm{AuPd} / \mathrm{BiVO}_{4}$ composites with different weight addition ratios of AuPd bimetallic NPs.

Fig. 2. SEM images of pure $\mathrm{BiVO}_{4}$ (a), AuPd/BiVO 4 (b), TEM image (c), HRTEM image (d) and EDX spectra (e) of the AuPd/BiVO 4 sample.

Fig. 3. TEM image of $\mathrm{AuPd} / \mathrm{BiVO}_{4}$ at low magnification (a); elemental distribution mapping of (b) O,

(c) Pd, (d) Au, (e) V and (f) Bi.

Fig. 4. UV-visible diffuse reflectance spectra of pure $\mathrm{BiVO}_{4}$ nanosheets, $\mathrm{Au} / \mathrm{BiVO}_{4}, \mathrm{Pd} / \mathrm{BiVO}_{4}$ and $\mathrm{AuPd} / \mathrm{BiVO}_{4}$ composites with different weight addition ratios of AuPd NPs.

Fig. 5. XPS spectra of AuPd/BiVO 4 samples: (a) Bi 4f; (b) V 2p; (c) O 1s; (d) Au 4f ;(e)Pd 3d.

Fig. 6. The photodegradation curves of $\mathrm{RhB}$ over different photocatalyst: pure $\mathrm{BiVO}_{4}(\mathrm{~A}), 1.0 \mathrm{wt} \%$ AuPd/BiVO 4 (A-1), 0.5 wt\% AuPd/BiVO 4 (A-2), 3wt\% AuPd/BiVO 4 (A-3), 5.0 wt\% AuPd/BiVO (A-4), 1.0 wt\% $\mathrm{Pd} / \mathrm{BiVO}_{4}$ (A-5), 1.0 wt\% $\mathrm{Au} / \mathrm{BiVO}_{4}$ (A-6) under visible-light irradiation.

Fig. 7. (a) The first-order kinetics of RhB degradation of different photocatalyst $\mathrm{BiVO}_{4}(\mathrm{~A}), 1$ wt $\%$ $\mathrm{AuPd} / \mathrm{BiVO}_{4}$ (A-1), $0.5 \mathrm{wt} \% \mathrm{AuPd} / \mathrm{BiVO}_{4}$ (A-2), $3.0 \mathrm{wt} \% \mathrm{AuPd} / \mathrm{BiVO}_{4}$ (A-3), $5.0 \mathrm{wt} \% \mathrm{AuPd} / \mathrm{BiVO}_{4}$ (A-4), $1.0 \mathrm{wt} \% \mathrm{Pd} / \mathrm{BiVO}_{4}$ (A-5), $1.0 \mathrm{wt} \% \mathrm{Au} / \mathrm{BiVO}_{4}$ (A-6) under visible light irradiation. (b) The degradation rate constant of RhB with different samples.

Fig. 8. The photodegradation curves (red line) of RhB dye, and the phenol photo-decomposition curve (blue line) over $1.0 \mathrm{wt} \% \mathrm{AuPd} / \mathrm{BiVO}_{4}$ sample under visible irradiation.

Fig. 9. Cycling runs for the photocatalytic degradation of methyl orange in the presence of $1 w t \%$ $\mathrm{Pd} / \mathrm{BiVO}_{4}$ composite under visible light irradiation.

Fig. 10. ESR spectra record at ambient temperature with $1.0 \mathrm{wt} \% \mathrm{AuPd} / \mathrm{BiVO}_{4}$ photocatalyst in: (a) methanol; (b) $\mathrm{H}_{2} \mathrm{O}$. 
Fig. 11. The photodegradation curves of RhB (black line) and the effect of scavenger KI (red line).

Fig. 12. SPV spectra of the pure $\mathrm{BiVO}_{4}, 1.0 \mathrm{wt} \% \mathrm{AuPd} / \mathrm{BiVO}_{4}, 1.0 \mathrm{wt} \% \mathrm{Pd} / \mathrm{BiVO}_{4}$ and $1.0 \mathrm{wt} \%$ $\mathrm{Au} / \mathrm{BiVO}_{4}$

Fig. 13. Schematic diagram illustrating the model of $\mathrm{AuPd} / \mathrm{BiVO}_{4}$ production and the proposed degradation mechanism of $\mathrm{RhB}$ over $\mathrm{AuPd} / \mathrm{BiVO}_{4}$ composites. 
1.

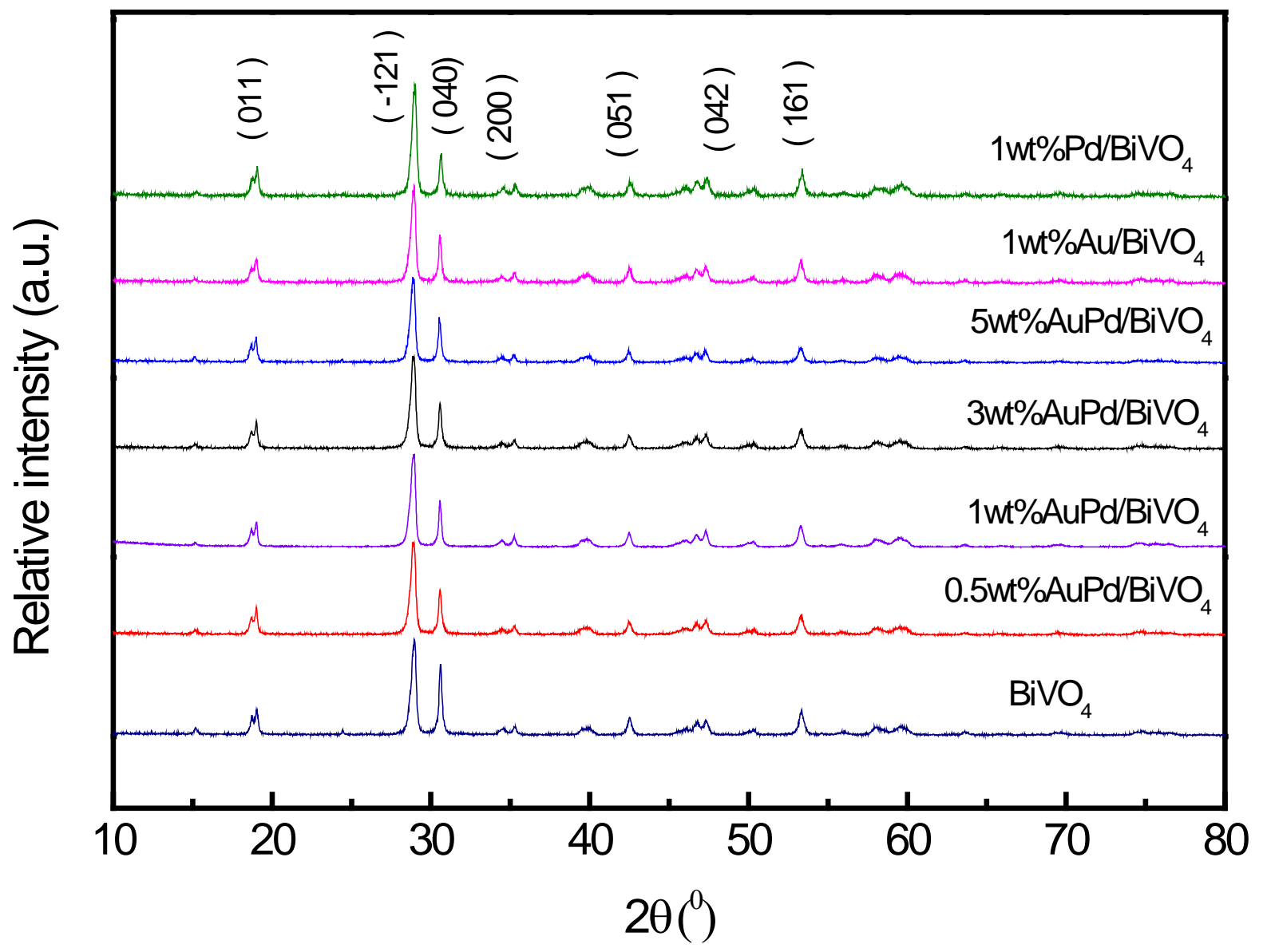

Fig. 1. XRD patterns of pure $\mathrm{BiVO}_{4}$ nanosheets, $\mathrm{Au} / \mathrm{BiVO}_{4}, \mathrm{Pd} / \mathrm{BiVO}_{4}$ and $\mathrm{AuPd} / \mathrm{BiVO}_{4}$ composites with different weight ratios of AuPd bimetallic NPs. 
2.
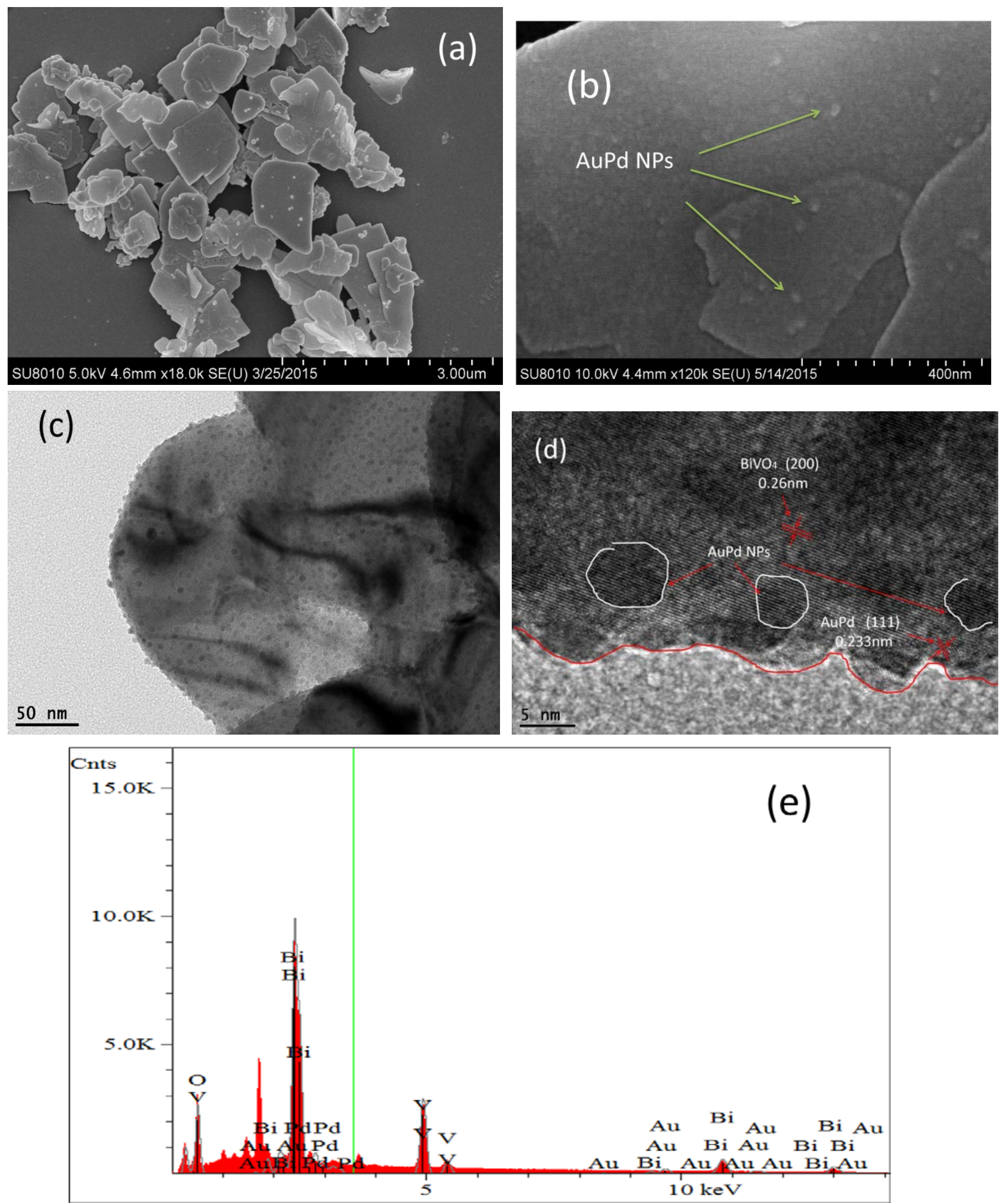

Fig. 2. SEM images of pure $\mathrm{BiVO}_{4}$ (a), AuPd/BiVO 4 (b), TEM image (c), HRTEM image (d) and EDX spectra (e) of the AuPd/BiVO 4 sample. 
3.

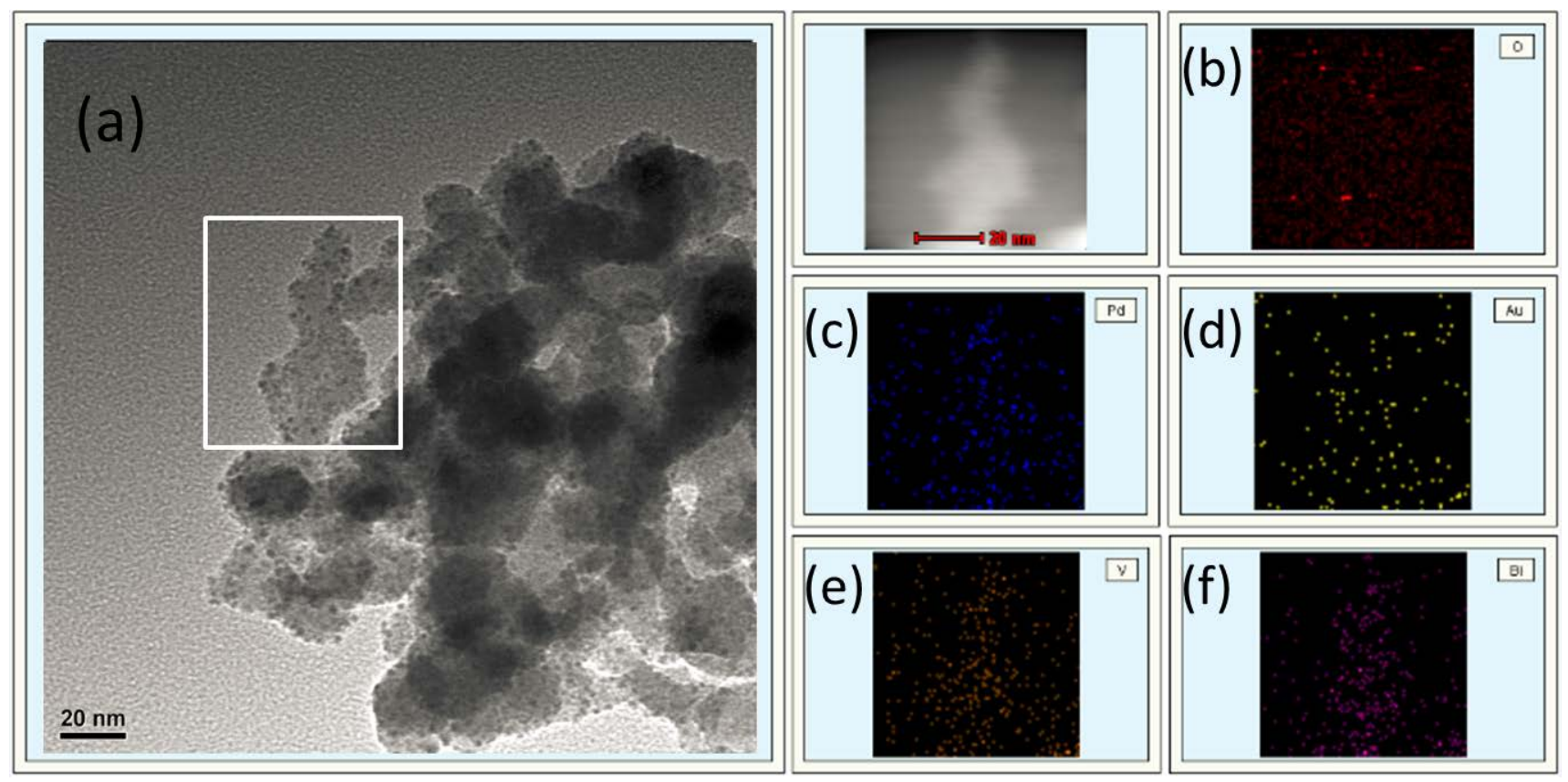

Fig. 3. TEM image of AuPd/BiVO 4 at low magnification (a); elemental distribution mapping of (b) O, (c) Pd, (d) Au, (e) V and (f) Bi. 
4.

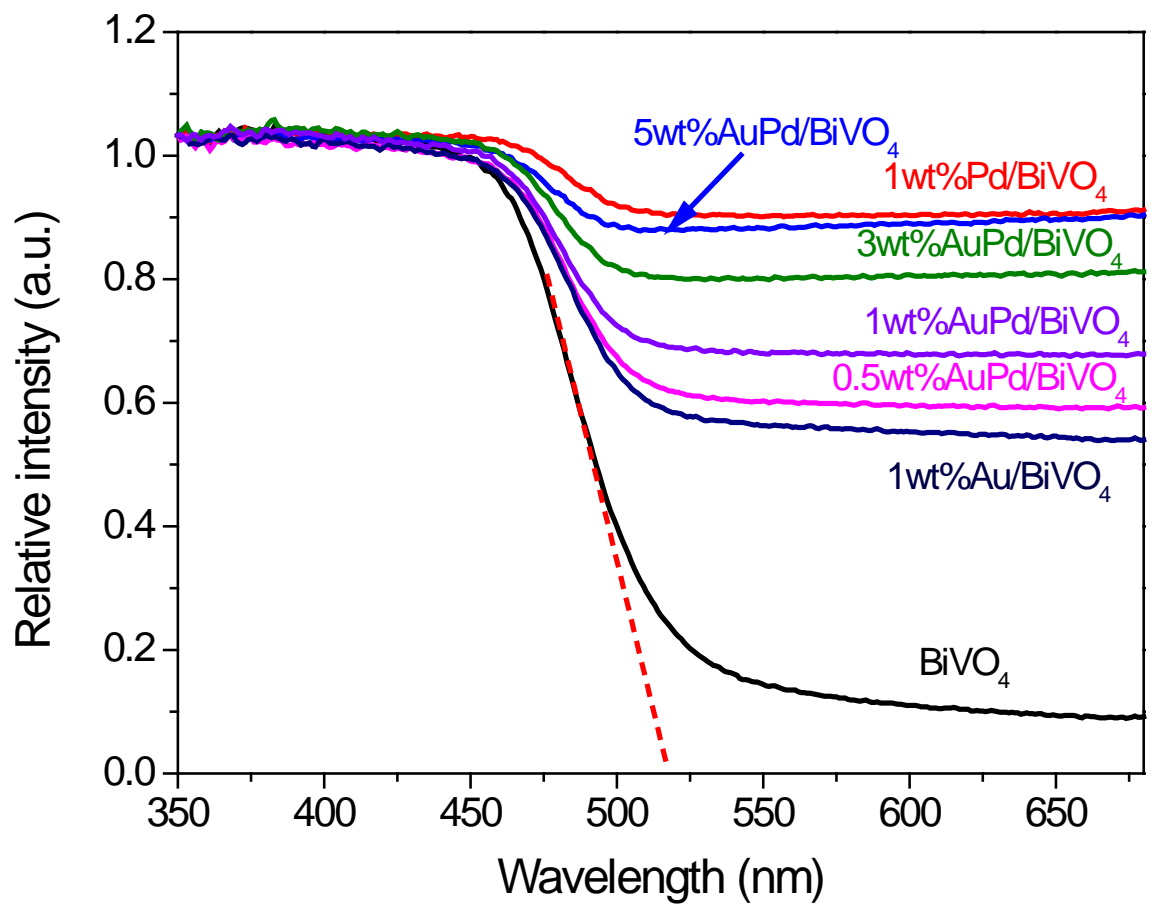

Fig. 4. UV-visible diffuse reflectance spectra of pure $\mathrm{BiVO}_{4}$ nanosheets, $\mathrm{Au} / \mathrm{BiVO}_{4}, \mathrm{Pd} / \mathrm{BiVO}_{4}$ and $\mathrm{AuPd} / \mathrm{BiVO}_{4}$ composites with different weight ratios of AuPd NPs. 
5.
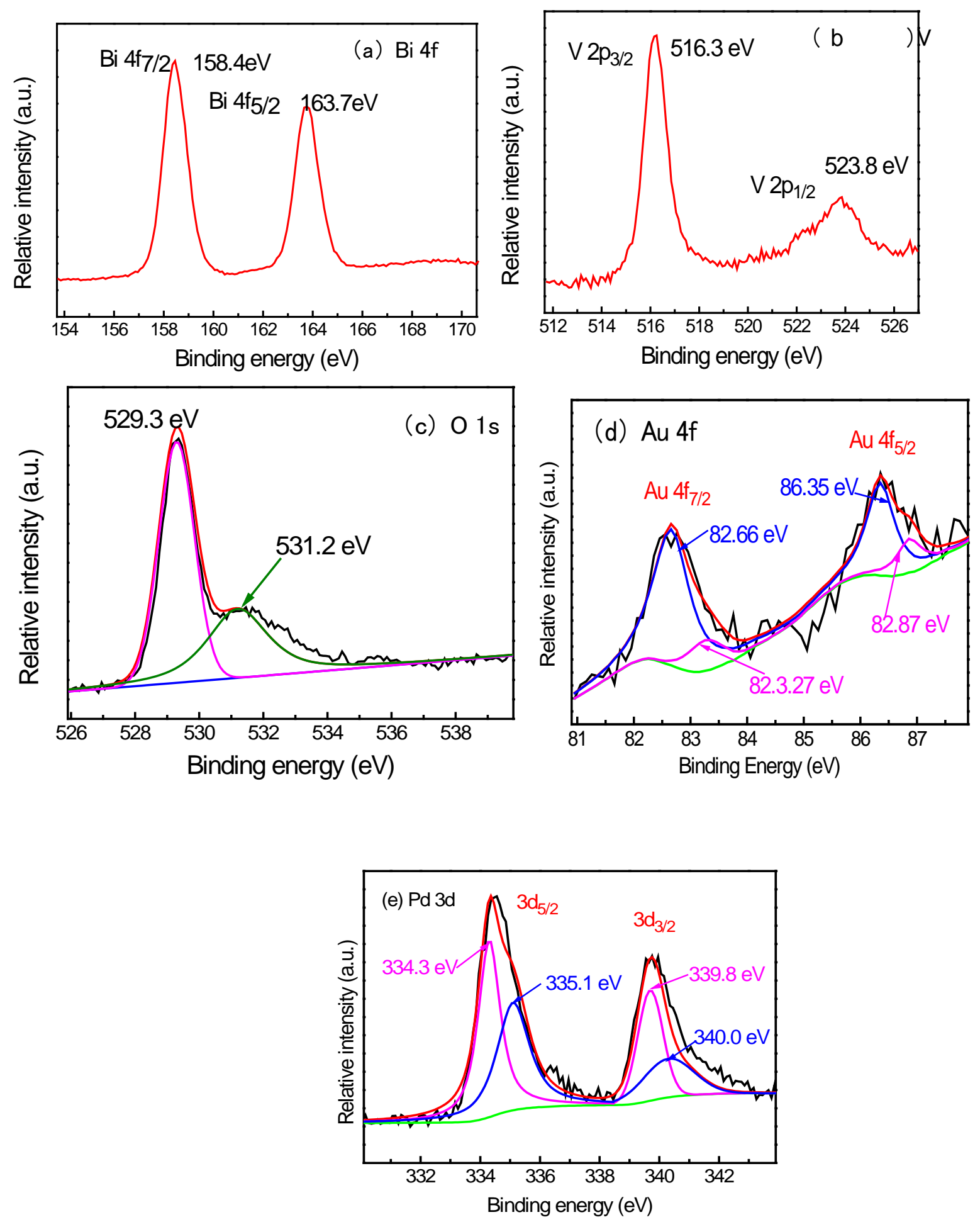

Fig. 5. XPS spectra of AuPd/BiVO 4 samples: (a) Bi 4f; (b) V 2p; (c) O 1s; (d) Au 4f ;(e)Pd 3d. 


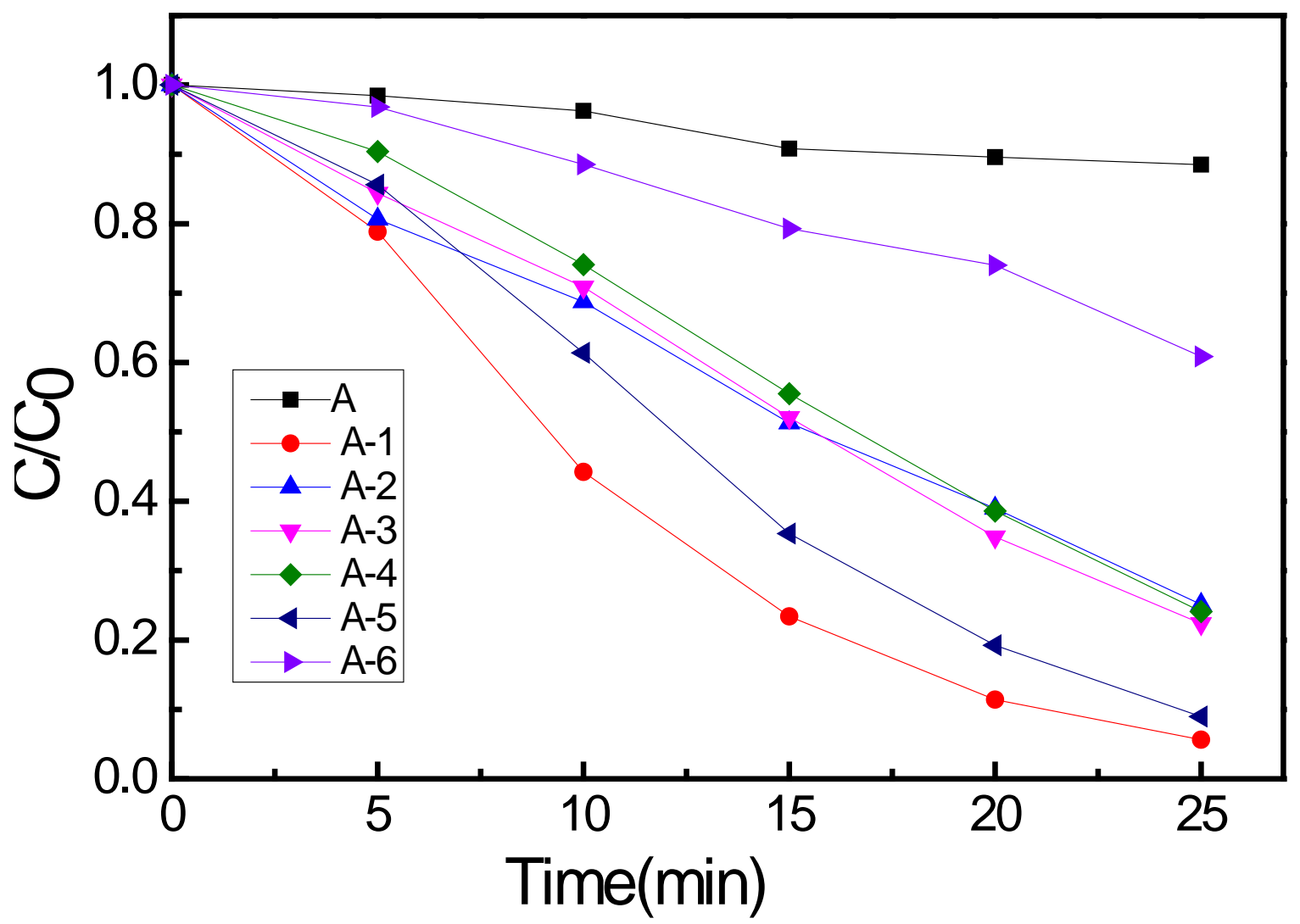

Fig. 6. The photodegradation curves of RhB over different photocatalyst: pure $\mathrm{BiVO}_{4}(\mathrm{~A}), 1.0 \mathrm{wt} \%$ AuPd/BiVO 4 (A-1), 0.5 wt\% AuPd/BiVO 4 (A-2), 3wt\% AuPd/BiVO 4 (A-3), 5.0 wt\% AuPd/BiVO 4 (A-4), $1.0 \mathrm{wt} \% \mathrm{Pd} / \mathrm{BiVO}_{4}$ (A-5), $1.0 \mathrm{wt} \% \mathrm{Au} / \mathrm{BiVO}_{4}$ (A-6) under visible-light irradiation. 
7.
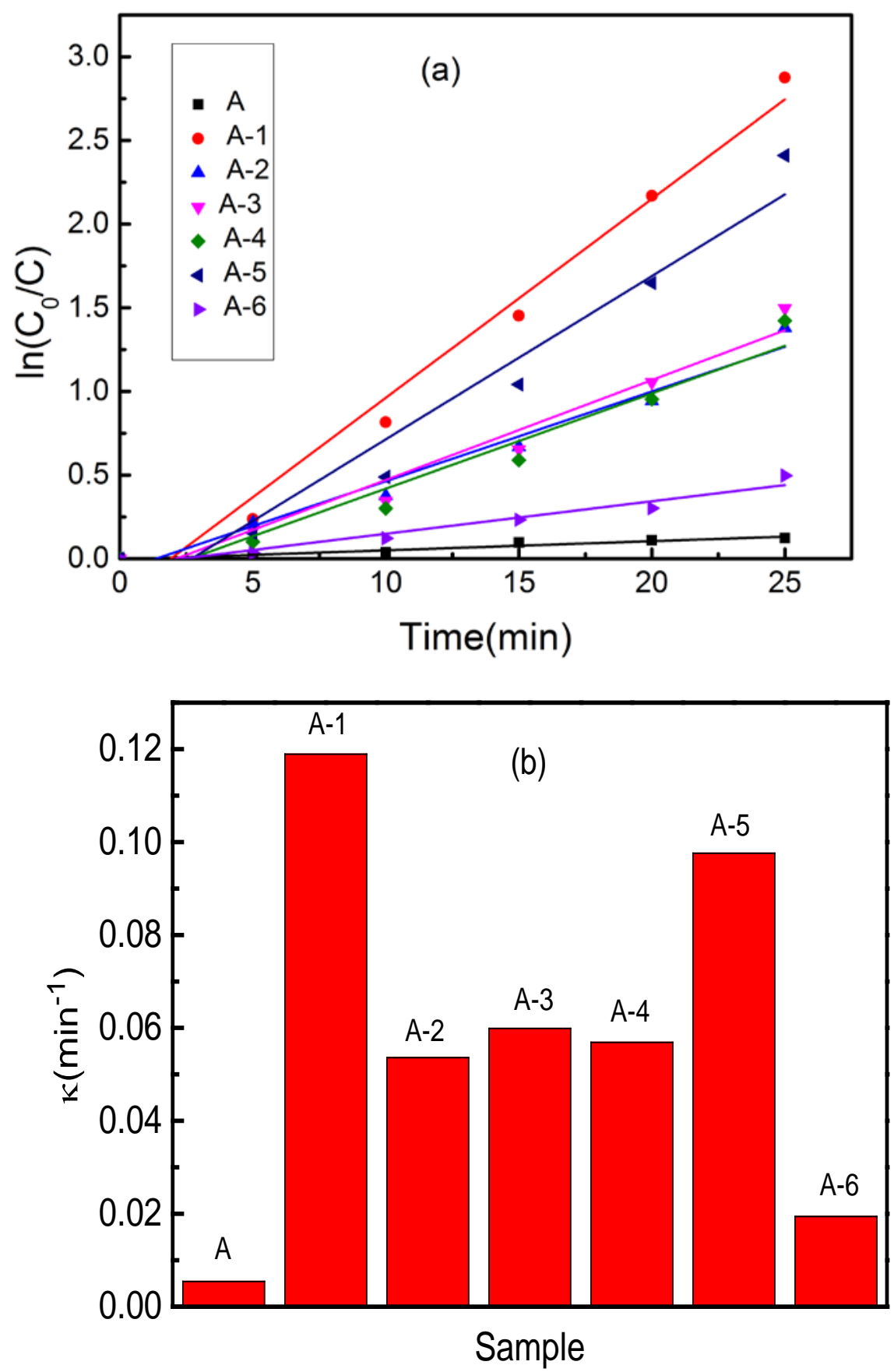

Fig. 7. (a) The first-order kinetics of RhB degradation of different photocatalyst $\mathrm{BiVO}_{4}$ (A), 1 wt $\%$ AuPd/BiVO 4 (A-1), 0.5 wt\% AuPd/BiVO 4 (A-2), 3.0 wt\% AuPd/BiVO 4 (A-3), 5.0 wt\% AuPd/BiVO 4 (A-4), $1.0 \mathrm{wt} \% \mathrm{Pd} / \mathrm{BiVO}_{4}$ (A-5), $1.0 \mathrm{wt} \% \mathrm{Au} / \mathrm{BiVO}_{4}$ (A-6) under visible light irradiation. (b) The degradation rate constant of $\mathrm{RhB}$ with different samples. 
8.

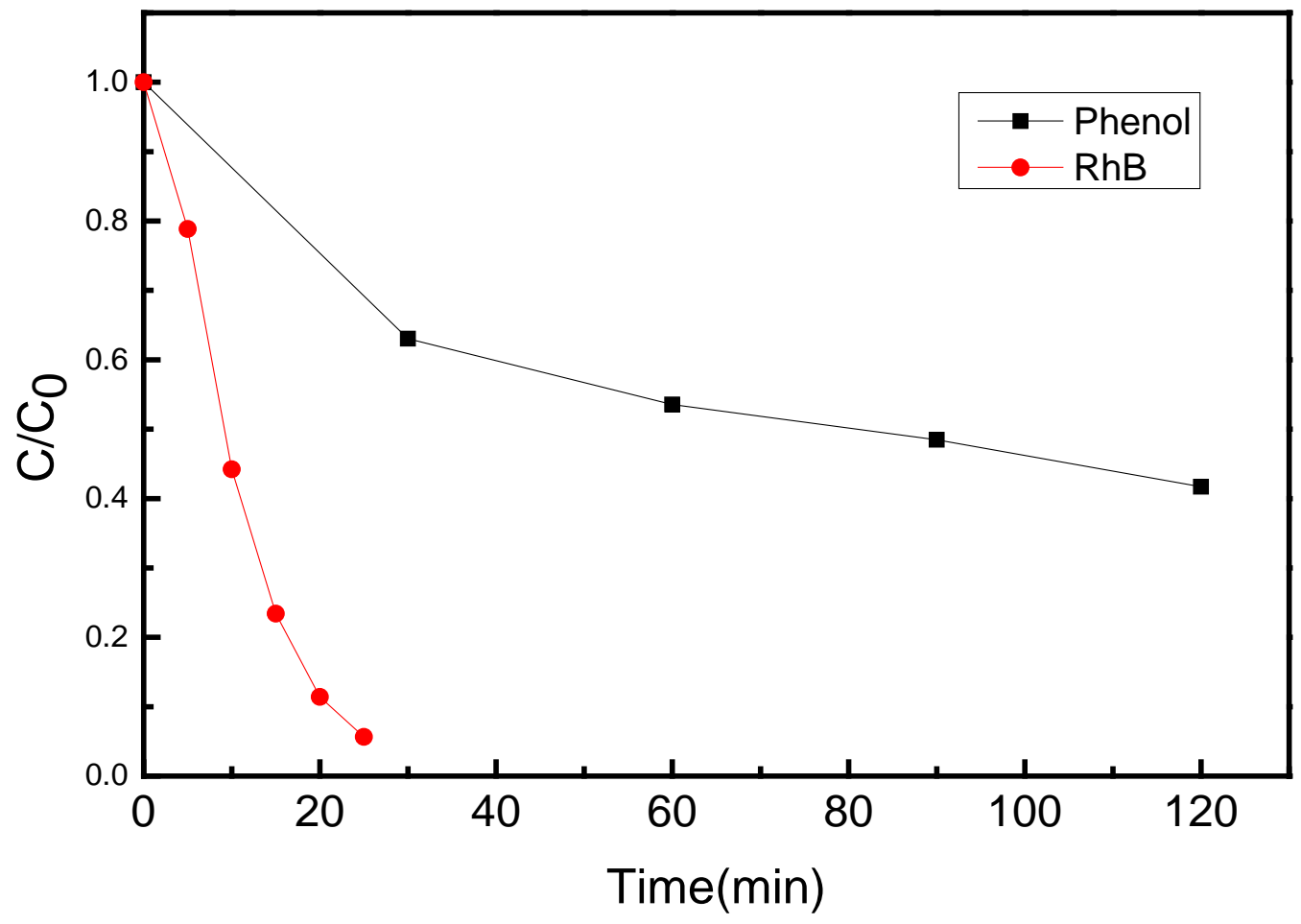

Fig. 8. The photodegradation curves (red line) of RhB dye, and the phenol photo-decomposition curve (blue line) over $1.0 \mathrm{wt} \% \mathrm{AuPd} / \mathrm{BiVO}_{4}$ sample under visible irradiation. 
9.

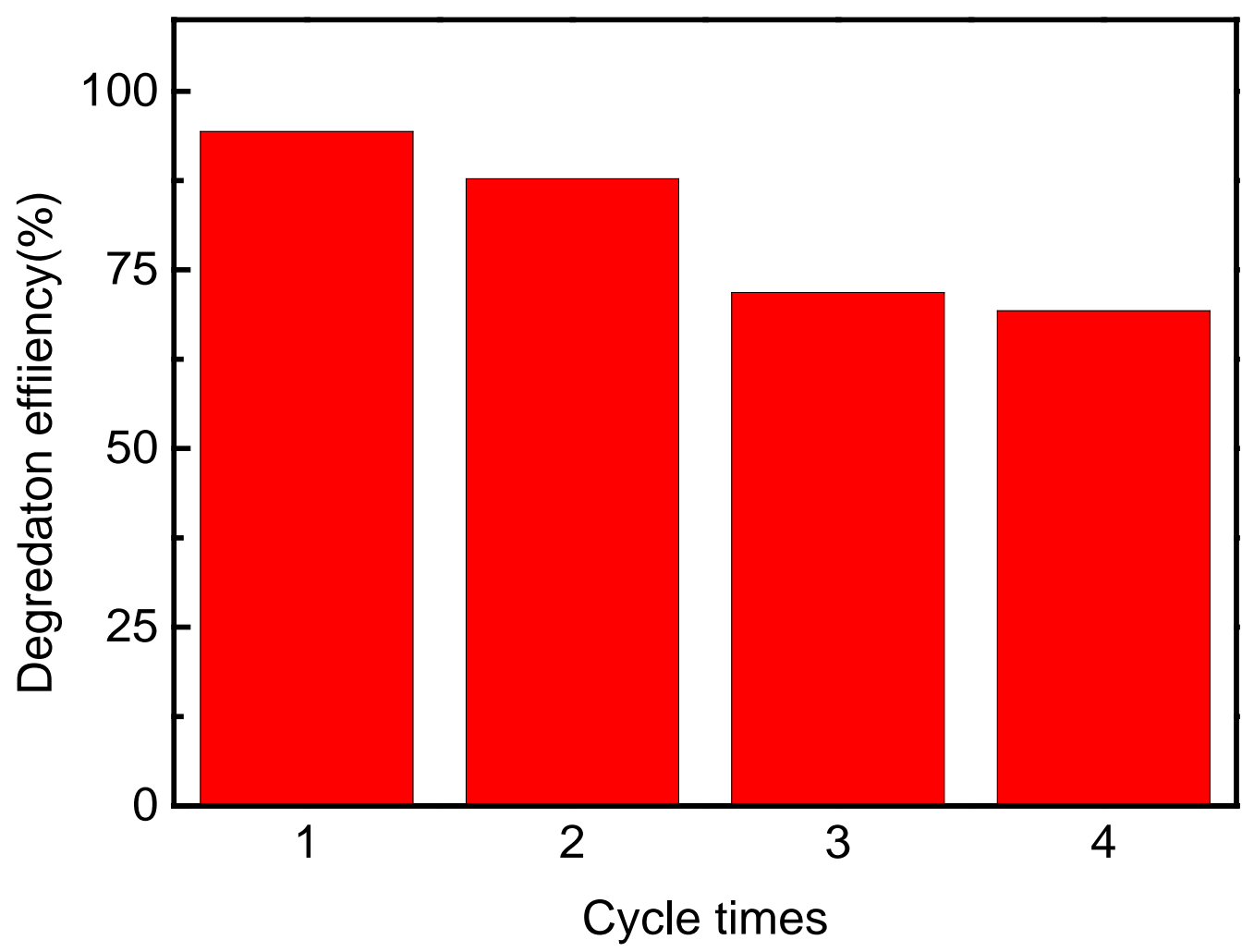

Fig. 9. Cycling runs for the photocatalytic degradation of methyl orange in the presence of $1 \mathrm{wt} \%$ $\mathrm{Pd} / \mathrm{BiVO}_{4}$ composite under visible light irradiation. 
10.
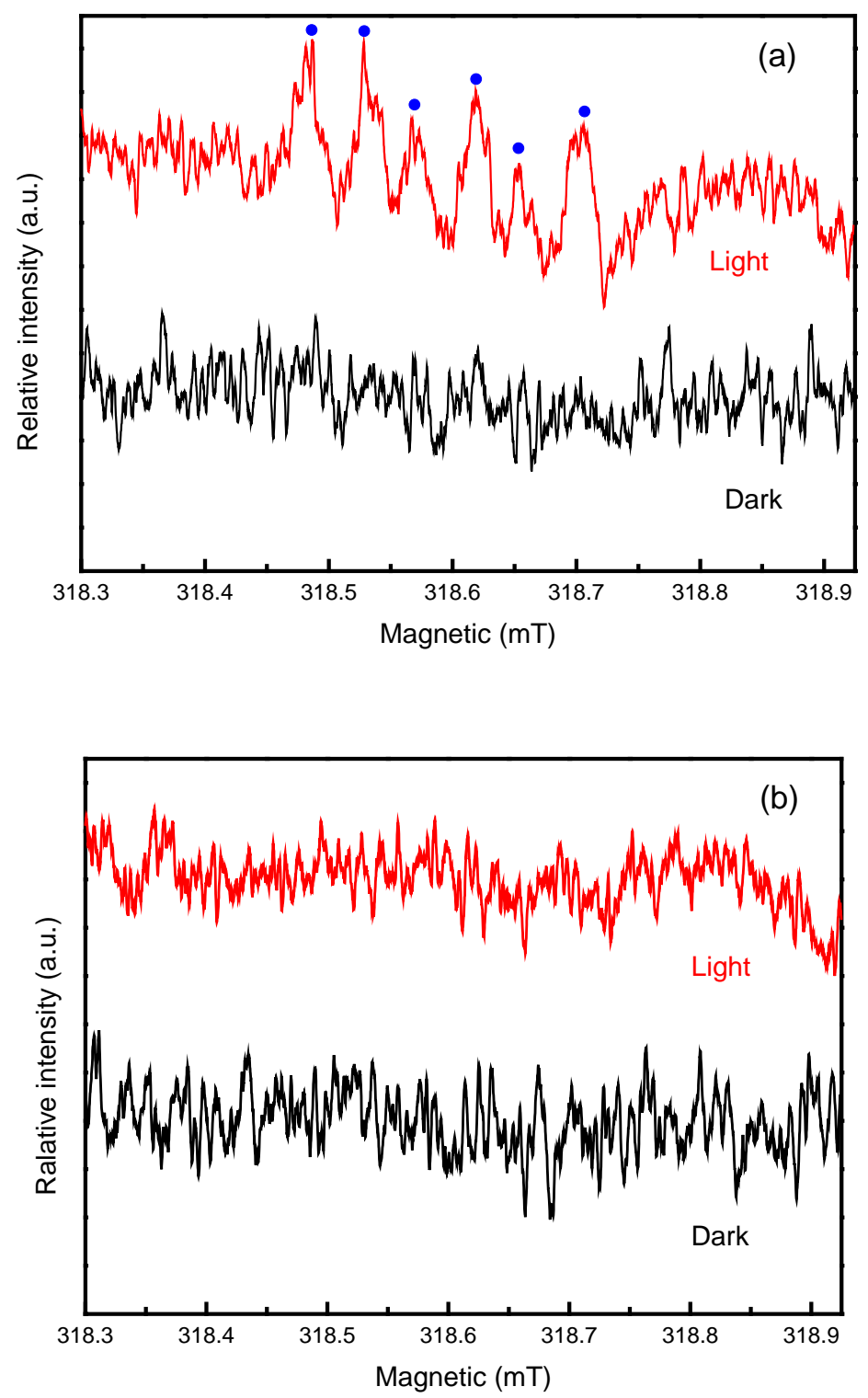

Fig. 10. ESR spectra record at ambient temperature with $1.0 \mathrm{wt} \% \mathrm{AuPd} / \mathrm{BiVO}_{4}$ photocatalyst in : (a) methanol;(b) $\mathrm{H}_{2} \mathrm{O}$. 
11.

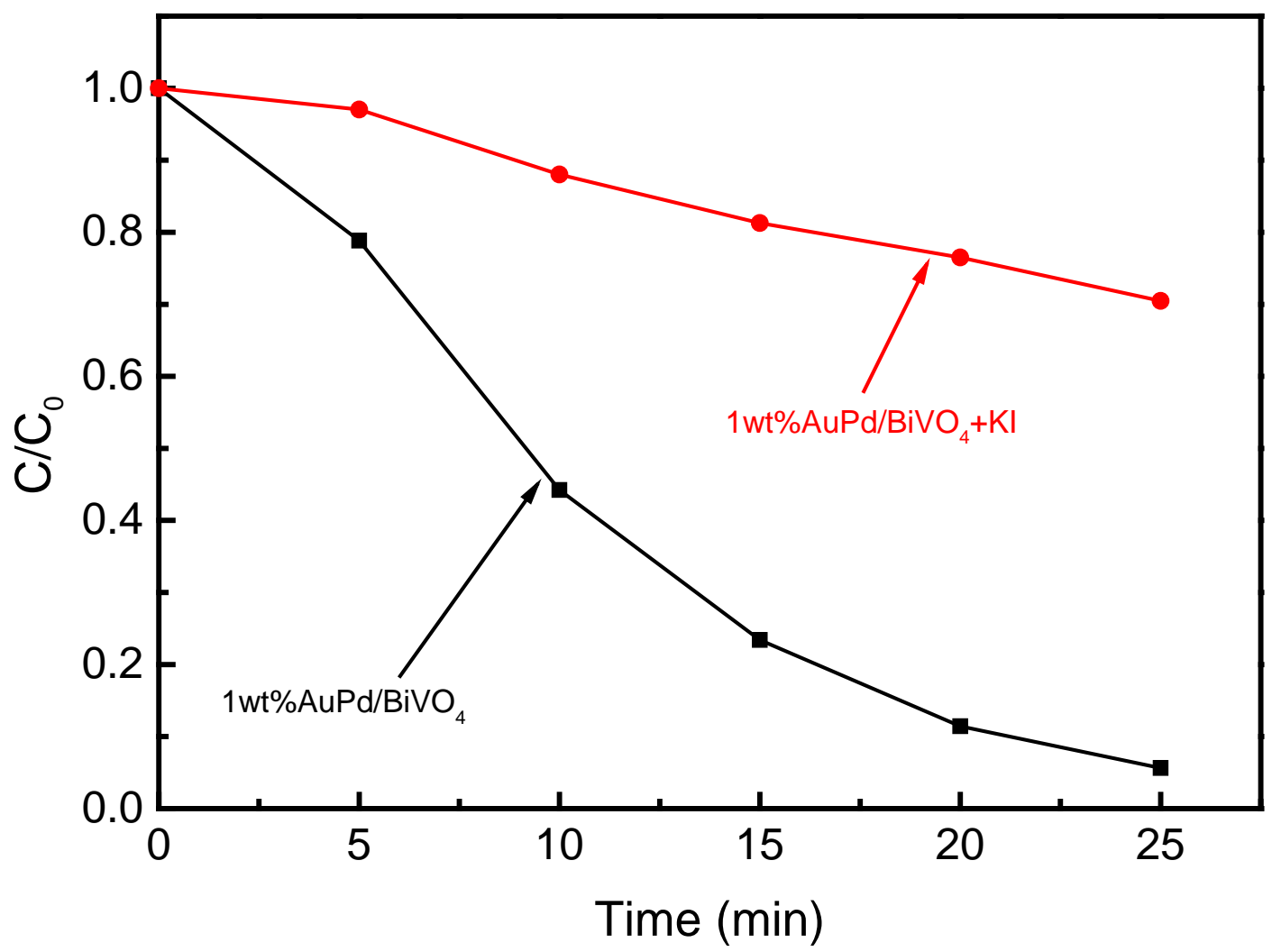

Fig. 11. The photodegradation curves of RhB (black line) and the effect of scavenger KI (red line). 
12.

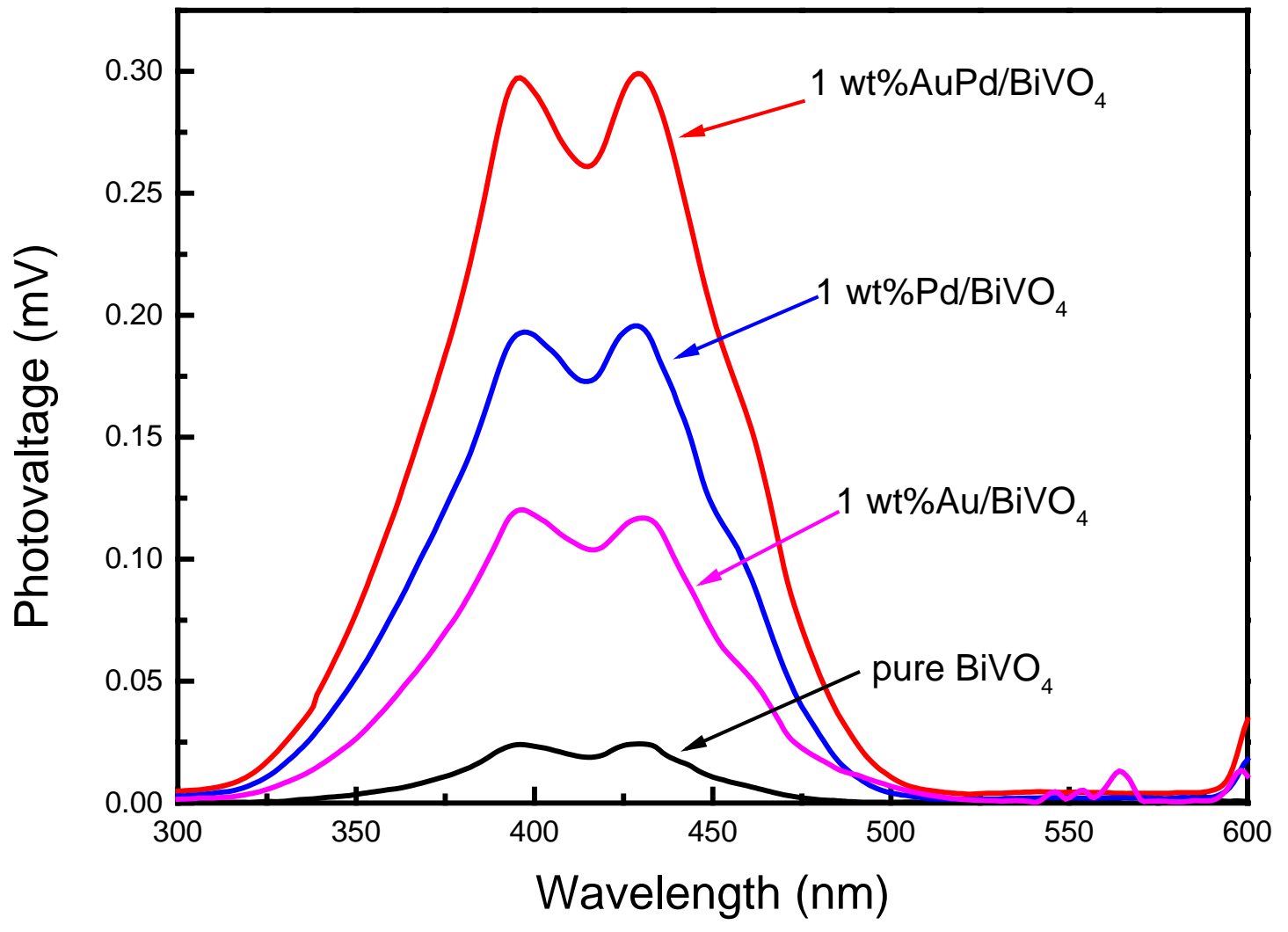

Fig. 12. SPV spectra of the pure $\mathrm{BiVO}_{4}, 1.0 \mathrm{wt} \% \mathrm{AuPd} / \mathrm{BiVO}_{4}, 1.0 \mathrm{wt} \% \mathrm{Pd} / \mathrm{BiVO}_{4}$ and $1.0 \mathrm{wt} \%$ $\mathrm{Au} / \mathrm{BiVO}_{4}$ 
13.

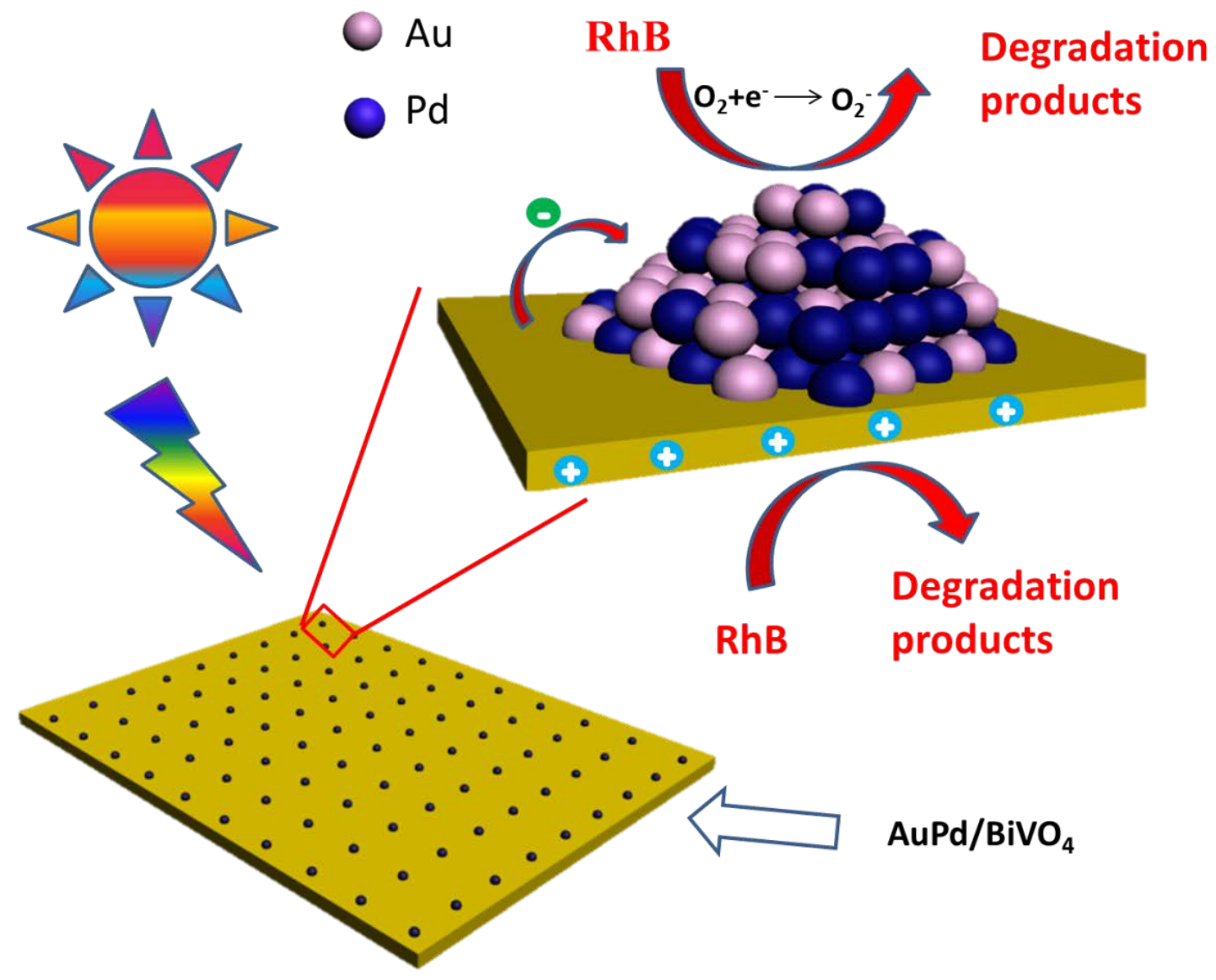

Fig. 13. Schematic diagram illustrating the model of $\mathrm{AuPd} / \mathrm{BiVO}_{4}$ production and the proposed degradation mechanism of $\mathrm{RhB}$ over $\mathrm{AuPd} / \mathrm{BiVO}_{4}$ composites. 